\title{
Path planning of Robot Based on Ant Colony Algorithm
}

\author{
Kai Jiang ${ }^{1}$, Chungui $\mathrm{Li}^{2}$ \\ ${ }^{1}$ School of Electrical and Information Engineering, Guangxi University of Science and Technology, \\ Liuzhou, Guangxi, China \\ ${ }^{2}$ School of Computer Science, Guangxi University of Science and Technology, Liuzhou, Guangxi, \\ China \\ 1jiangkai0516@126.com
}

Keywords: Robot; Path planning; Ant Colony Algorithm

\begin{abstract}
With the development of the science and technology, intelligent robotic systems have been applied in the service industry, and the R\&D of service-type autonomous mobiles robot has received greater attention from business circles in china. Now, many combination optimization algorithms have been developed to solve the robot path planning, but with some limitation for each algorithms. The newly-developed and algorithm has superiorities of positive feedback, flexibility and collaboration, which enables it to adapt to the trend of path planning algorithms in the intelligent and bionic direction. Thus, ant colony algorithm is proposed to plan the robot's path in this paper.
\end{abstract}

\section{Introduction}

Robot path planning is an important research field. It requires that the robot based on one or some of the optimization principle (such as minimum energy loss shortest, the shortest routes, the short of walking time, etc.), then to find a path from the initial state to the target state can avoid most obstacles in their work space path. Robot path planning problem can be modeled as a constrained optimization problem, we must complete the path planning, positioning and obstacle avoidance tasks.

Ant colony algorithm is a novel simulated evolutionary algorithm that simulate the natural foraging behavior of real ant colony formed, the algorithm is first proposed by Italy scholar M.Dorigo. In recent years, has also been introduced into the path planning. The design of the new algorithm, the main characteristics depend that is the distance from point to away the target point, the point visit times and direction of movement pheromone called heuristic factor to guide artificial ants to find an optimal solution.

The main discuss that path planning of robot use of ant colony algorithm. Static robot known as global environment, build a robot workspace model, and uses the ant colony algorithm to find an optimal or near-optimal path.

\section{Introduced model of robot based on ant colony algorithm}

Ant colony algorithm is by The Italian scholar M.Dorigo,V.Maniezzo,A.Colorni first proposed that ant colony algorithm is based on simulate the evolution of population, and a new heuristic algorithm by used to solve complex optimization problems in the 1990s.Principle is as follows:

Between individuals of ants through a substance of called pheromone have been information transfer. Ants have perceived this substance presence and intensity in movement, and ants tend to the high of substance intensity direction movement. Therefore, the more ants of walking a path, then latecomers choose the path the great probability. Among ants are through the exchange of information to achieve the purpose of search for food.

In order to simulate ants the real behavior, and we introduce the follow notation: m represents the number of ants in the colony, $d_{i j}$ represents the distance that from $i$ point to another $j$ point, $b_{i}(t)$ represents time $t$ is located in point $i$ the number of ants, $\tau_{i j}(t)$ represents at time $t$ the amount of information left in the ij connection. 
At the initial time, was equally the amount of information on each path, that is

$\tau_{\mathrm{ij}}(0)=\mathrm{C}(\mathrm{C}$ is a constant $)$.Ant $\mathrm{k}(\mathrm{k}=1,2, \ldots, \mathrm{m})$ accord that the amount of information on each path decided to shift direction in the process of movement.

$$
p_{i j}^{k}= \begin{cases}\frac{\tau_{i j}^{\alpha}(t) \eta_{i j}^{\beta}(t)}{\sum_{s \in \text { allowed }_{k}} \tau_{i s}^{\alpha} \eta_{i s}^{\beta}} & j \in \text { allowed }_{k} \\ 0 & \text { otherwise }\end{cases}
$$

Represents that ant $\mathrm{k}$ is transferred from position $\mathrm{i}$ to position $\mathrm{j}$ of the transition probability at time $t$. In the process of movement, $\alpha$ represents information of accumulation, $\beta$ represents the importance of the role heuristic factor in ants select the path. $\eta_{\mathrm{ij}}$ represents ank is transferred from point $\mathrm{i}$ to point $\mathrm{j}$ the desired degree, and take the reciprocal of the distance. allowed $_{\mathrm{k}}=\{0,1, \ldots, \mathrm{n}-1\}$-tabu $_{\mathrm{k}}$, this equation representation ant $\mathrm{k}$ allows to select the next point. Different with the actual ant, artificial ant colony system has a memory function. $\operatorname{tabu}_{\mathrm{k}}(\mathrm{k}=1,2, \ldots, \mathrm{m})$ records the ant $\mathrm{k}$ traversed points of the current. Set tabu $\mathrm{k}_{\mathrm{k}}$ with the process of evolution to make dynamic adjustments. With the passage of time,then it has been gradually disappeared before leaving the information. Parameters $1-\rho$ representation the degree of information disappearance. After $n$ hours, the ant have completed a cycle, and the information of each path conduct adjustment. For example formula 2 and formula 3:

$$
\begin{aligned}
\tau_{i j}(t+n) & =\rho * \tau_{i j}(t)+\Delta \tau_{i j} \quad \rho \in(0,1) \\
\Delta \tau_{i j} & =\sum_{k=1}^{m} \Delta \tau_{i j}^{k}
\end{aligned}
$$

The formula 3 indicates that the $\mathrm{k}$ ant stay on the path information in this cycle at the right,the left represents the incremental of information in this cycle on the path ij.

Update pheromone formula is selected as:

$$
\tau_{i j}(t+n)=(1-\rho) * \tau_{i j}(t)+\rho \Delta \tau_{i j} \quad \rho \in(0,1)
$$

The distance between the target point and the end point:

$$
D_{(\mathrm{i}, j)}=\sqrt{(1-N)^{2}+(j-N)^{2}}
$$

Probability of target point:

$$
Q_{(i, j)}=\frac{D_{(i, j)}^{-1} V_{(i, j)}^{-1} T_{(i, j)}}{\sum_{(x, y) \in N e x t^{(a, b)}} D_{(x, y)}^{-1} V_{(x, y)}^{-1} T_{(x, y)}}
$$

Among them: $\operatorname{Next}^{(a, b)}=\{(a-1, b),(a, b+1),(a+1, b),(a, b-1)\}$

We build a simulated environment according to their own requirements, for example

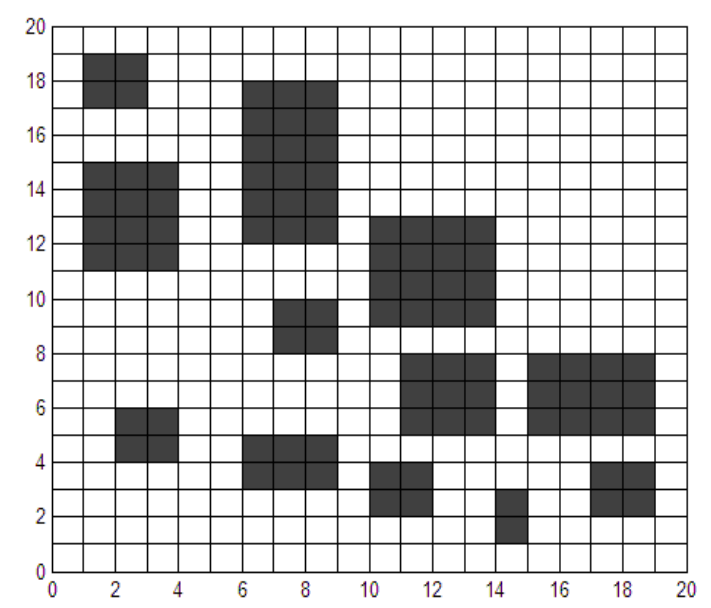

Fig.1 Static environment.

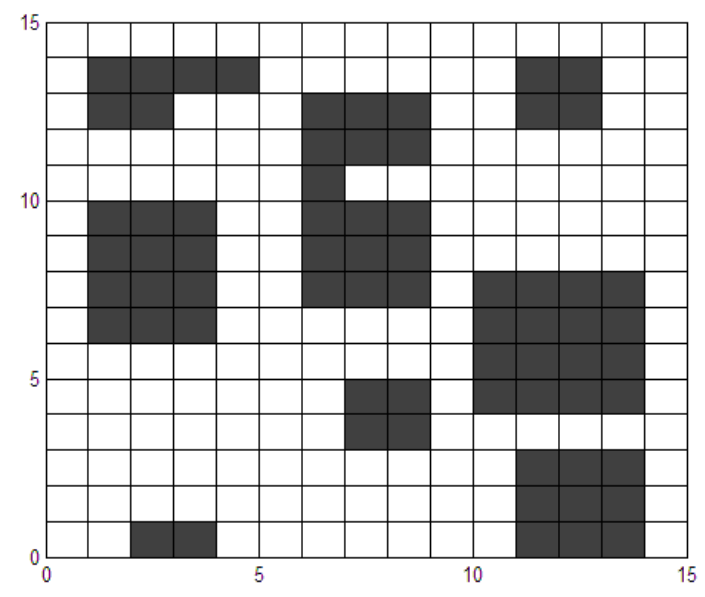

Fig.2 Static environment. 


\section{Algorithmic process}

We can complete the path planning of robot based on ant colony algorithm block diagram as shown below.

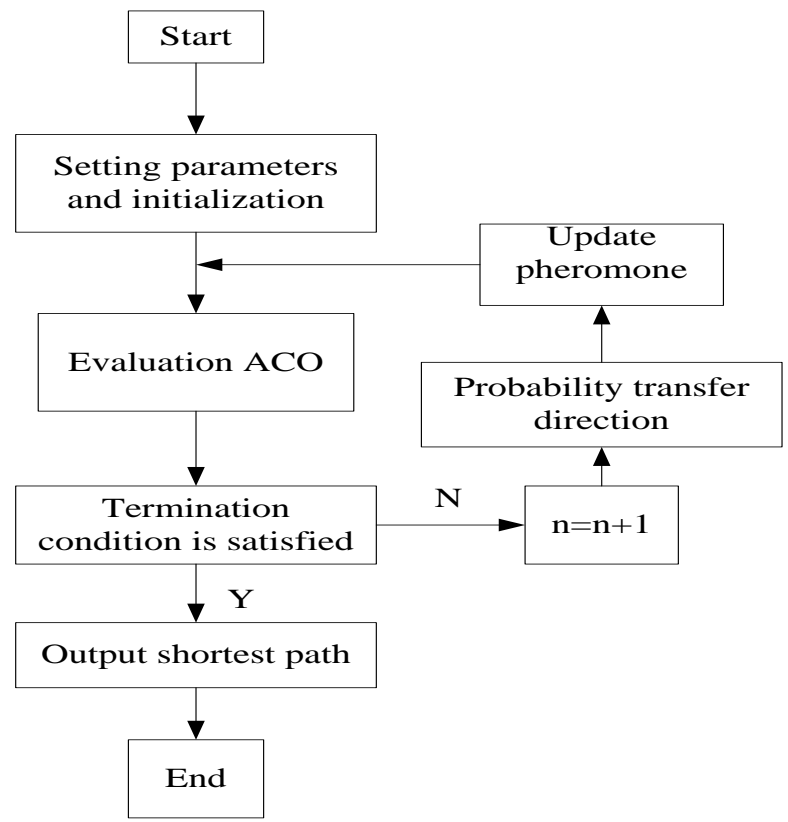

Fig.3 The flow chart of the ant colony algorithm

We want to build an environment model, for example:

Step 1 Initialization.

Step 2 while (Ants does not encounter food or into an infinite loop.)

Step 3 for $i=1$ to Number of ants

Step 4 Ant i choose to use probability function formula (6) to move to the next target point.

Step 5 Write down each ant of each generation foraging routes and route length.

Step 6 Update pheromone

if (Ants find a viable solution) then(Record, update the point to be accessed the number of times.) else(Ants death).

Step 7 According to the formula(4) in order to the optimal solution update the pheromone of path, the output of the optimal solution.

Multi steps solving: can be set the optimal solution, after iterative $m$ times has not been progress as the convergence of the algorithm.

\section{Experimental simulation results}

Graphic simulation of the first model.

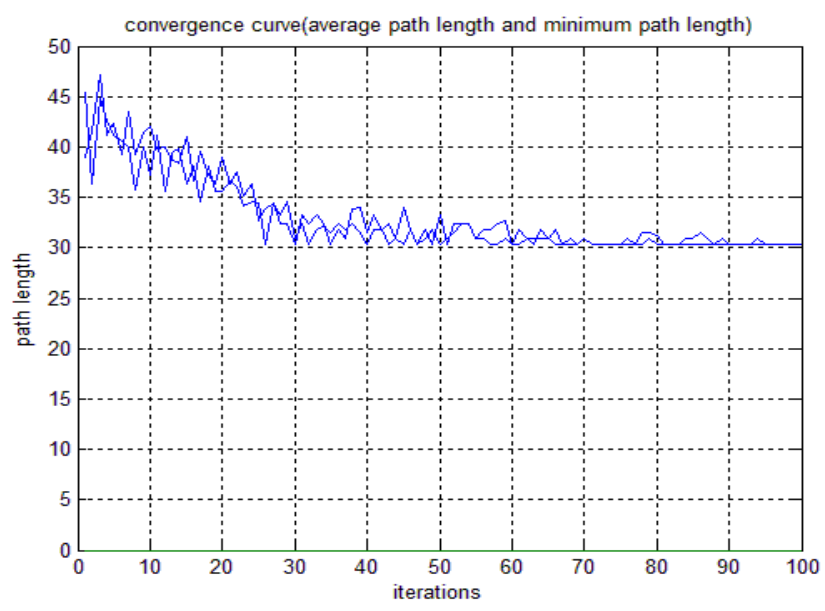

Fig.4 Two iterative curves. 


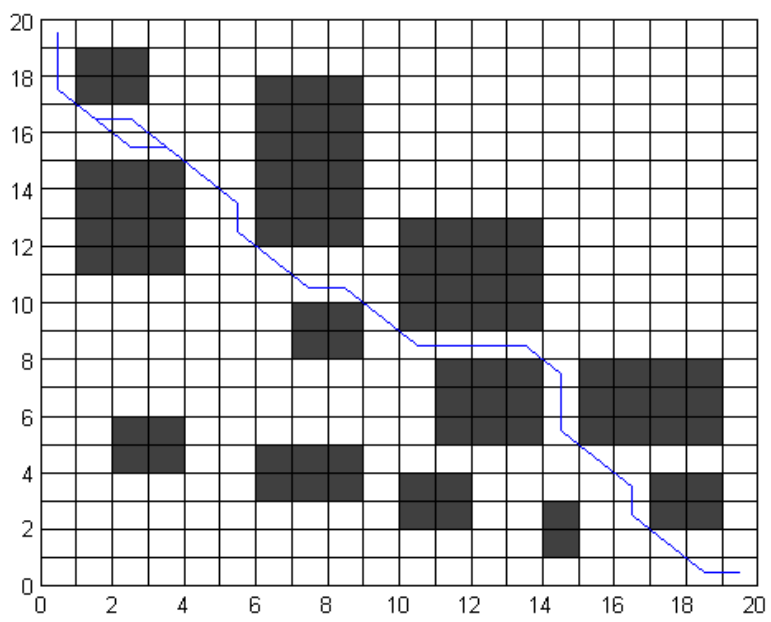

Fig.5 Path planning diagram.

Graphic simulation of the second model.

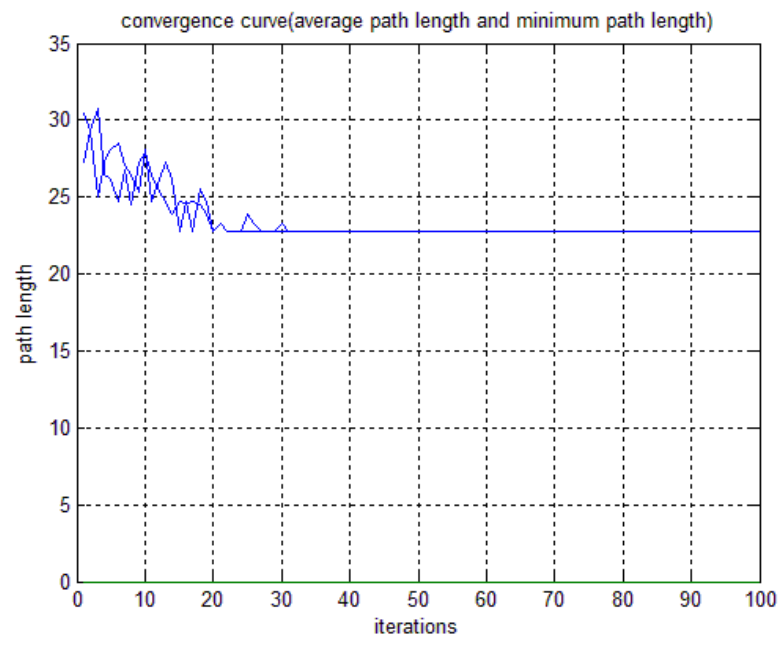

Fig.6 Two iterative curves.

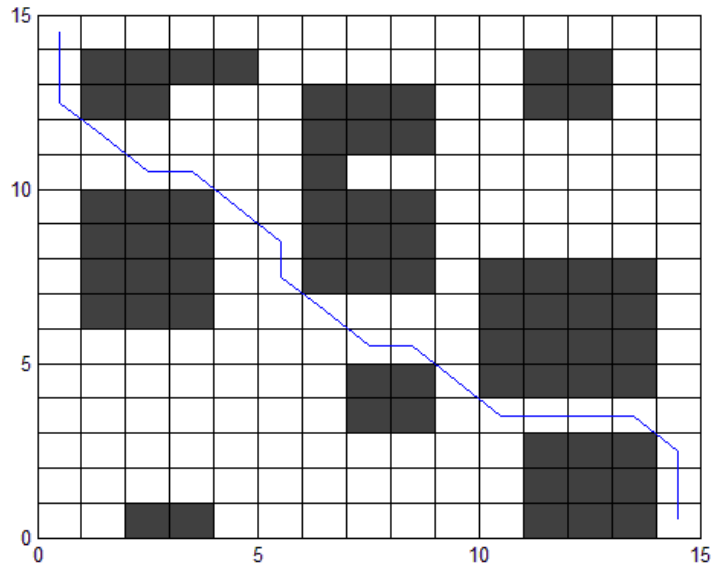

Fig.7 Path planning diagram.

Analysis of experimental results: On the Matlab platform, the simulation result shows that the optimal path can be built quickly by this algorithm if the environment map is known. Also, the algorithm is simple and efficient. Comparison with traditional search algorithms-the robot can find the goal within the shortest path without the collision and the stagnation. We can see two iterative curves along the line in Figure 4, then the result is the same distance on the image (Figure 5). We can see that have a parallelogram is obtained of two iterative curve in Figure 5, but the length of the line is the same. According to the second environment model that can be drawn graphic simulation 
is very correct. After two iterative curves (figure 6), the line is only one in figure 7. Therefore, it's taking the route shortest and optimal so as to arrive the algorithm is very stable.

\section{Summary}

Ant colony algorithm is a random search optimization method from nature. Now, it has been applied to various fields of combinatorial optimization, communication and others. We can compare ant colony algorithm with traditional genetic algorithms and simulated annealing, which has better adaptability. Positive feedback, collaborative and implicit parallelism so that it has a strong potential for development of the ant colony algorithm. Ant colony algorithm has strong flexibility so that make to solve combinatorial optimization problems with good adaptability. Therefore, the ant colony algorithm is applied to study the intelligent robot to avoid obstacles in the path planning problem. It is able to explore and improve a new path optimization algorithm, and promote optimization theory or development of practice, then provide a reference for the economy field and engineering field optimization problems.

\section{Reference}

[1] Marco Dorigo, Gianni Di Caro, Luca M GambardellaAnt algorithms for Discrete Optimization. Massachusetts Institute of Technology, Artificial Life, 1999: 137-172.

[2] Dorigo M.Maniezzo V,Colomi A.Ant System: Optimization by A Colony of Cooperating Agents[J]. IEEE Transactions on System, Man, and Cybemetics,Partt B,1996;26(1):28-41.

[3] Thomas Stutzle, Holger Hoos. MAX-MIN Ant System. Future Generation system. 2000; (16): $889 \sim 914$.

[4] Kazuo Sugibara, John Smith.Gmrtic Algorithms for Adaptive Motion Planning of an Autonomous Mobile Robots[C].In:Problems IEEE Trans.SMC,MIT,US,1997:138-143.

[5] Pedro I niguez.Jan Rosell E.cient Path Planning Using Harmonic Functions Computed on a Non-regular Grid[C].In:M T Escrig Monferer and F Toledo Lobo Eds,CC1A 2002,LNAI 2504,2002:345-354.

[6] Clare Davies,Eric Pederson. Grid Patterns and Cultural Expectations in Urban Wayfinding[C]. In: D R Montello Ed. COSIT 2001, LNCS 2205, 2001: 400-414.

[7] Peter Yap,Grid-Based Path-Finding,R Cohen et a1.AI 2002,LNAI 2338,2002:44-55.

[8] M B M etea.Route planning for intelligent autonomous land vehicles using hierarchical terrain representation[C].In:Prnc of IEEE Int Conf on Robotics and Automation,1987:1947-1952. 